\title{
Estimated County-Level Prevalence of Selected Underlying Medical Conditions Associated with Increased Risk for Severe COVID-19 Illness - United States, 2018
}

\begin{abstract}
Hilda Razzaghi, $\mathrm{PhD}^{1}$; Yan Wang, $\mathrm{PhD}^{2}$; Hua Lu, $\mathrm{MS}^{2}$; Katherine E. Marshall, $\mathrm{MPH}^{1}$; Nicole F. Dowling, $\mathrm{PhD}^{1}$; Gabriela Paz-Bailey, MD, PhD ${ }^{1}$; Evelyn R. Twentyman, $\mathrm{MD}^{1}$; Georgina Peacock, $\mathrm{MD}^{1}$; Kurt J. Greenlund, $\mathrm{PhD}^{2}$
\end{abstract}

Risk for severe coronavirus disease 2019 (COVID-19)-associated illness (illness requiring hospitalization, intensive care unit [ICU] admission, mechanical ventilation, or resulting in death) increases with increasing age as well as presence of underlying medical conditions that have shown strong and consistent evidence, including chronic obstructive pulmonary disease, cardiovascular disease, diabetes, chronic kidney disease, and obesity (1-4). Identifying and describing the prevalence of these conditions at the local level can help guide decision-making and efforts to prevent or control severe COVID-19-associated illness. Below state-level estimates, there is a lack of standardized publicly available data on underlying medical conditions that increase the risk for severe COVID-19-associated illness. A small area estimation approach was used to estimate county-level prevalence of selected conditions associated with severe COVID-19 disease among U.S. adults aged $\geq 18$ years $(5,6)$ using self-reported data from the 2018 Behavioral Risk Factor Surveillance System (BRFSS) and U.S. Census population data. The median prevalence of any underlying medical condition in residents among 3,142 counties in all 50 states and the District of Columbia (DC) was $47.2 \%$ (range $=22.0 \%-66.2 \%)$; counties with the highest prevalence were concentrated in the Southeast and Appalachian region. Whereas the estimated number of persons with any underlying medical condition was higher in population-dense metropolitan areas, overall prevalence was higher in rural nonmetropolitan areas. These data can provide important local-level information about the estimated number and proportion of persons with certain underlying medical conditions to help guide decisions regarding additional resource investment, and mitigation and prevention measures to slow the spread of COVID-19.

BRFSS is an annual, random-digit-dialed landline and mobile telephone survey of noninstitutionalized U.S. adults aged $\geq 18$ years in all 50 states, DC, and U.S. territories. BRFSS collects self-reported information on selected health behaviors and conditions. Overall, 437,500 persons participated in the 2018 BRFSS survey, with a median weighted response rate of $49.9 \%$.*

*https://www.cdc.gov/brfss/index.html.

\section{INSIDE}

951 Identification of Substance-Exposed Newborns and Neonatal Abstinence Syndrome Using ICD-10-CM 15 Hospitals, Massachusetts, 2017

956 Evaluation of Online Risk Assessment To Identify Rabies Exposures Among Health Care Workers Utah, 2019

960 Population Point Prevalence of SARS-CoV-2 Infection Based on a Statewide Random Sample Indiana, April 25-29, 2020

965 Estimated Community Seroprevalence of SARS-CoV-2 Antibodies - Two Georgia Counties, April 28-May 3, 2020

971 Notes from the Field: Effects of the COVID-19 Response on Tuberculosis Prevention and Control Efforts - United States, March-April 2020

973 Notes from the Field: Characteristics of Tetrahydrocannabinol-Containing E-cigarette, or Vaping, Products Used by Adults - Illinois, September-October 2019

976 QuickStats

Continuing Education examination available at https://www.cdc.gov/mmwr/mmwr_continuingEducation.html

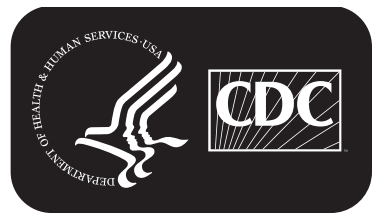

U.S. Department of Health and Human Services Centers for Disease Control and Prevention 
The underlying medical conditions included in these prevalence estimates were selected using the subset of the list of conditions with the strongest and most consistent evidence ${ }^{\dagger}$ of association with higher risk for severe COVID-19-associated illness on CDC's website as of June 25, 2020 (2) and for which questions on the BRFSS aligned. These included chronic obstructive pulmonary disease (COPD), heart conditions, diabetes mellitus, chronic kidney disease (CKD), and obesity (defined as body mass index [BMI] of $\geq 30 \mathrm{~kg}$ per $\mathrm{m}^{2}$ ). Conditions from the list of those with mixed and limited evidence ${ }^{\S}$ of association with increased risk for severe COVID-19 illness were not included (2). An analysis of U.S. COVID-19 patient surveillance data found that hospitalizations were six times higher, ICU admissions five times higher, and deaths 12 times higher among patients with underlying medical conditions, compared with those without (4); however, that analysis included a narrower definition of obesity (BMI $\geq 40 \mathrm{~kg}$ per $\mathrm{m}^{2}$ ), and some, but not all conditions in both the strongest and most consistent evidence and mixed and limited evidence lists.

\footnotetext{
$\dagger^{\dagger}$ Conditions with consistent evidence of increased risk for severe COVID-19associated illness from multiple small studies or a strong association from a large study.

$\$$ Conditions for which multiple studies have reached different conclusions about risk associated with that condition. Those with limited evidence are those for which consistent evidence has been reported from a small number of studies. https://www.cdc.gov/coronavirus/2019-ncov/need-extra-precautions/evidencetable.html.
}

BRFSS respondents were classified as having an underlying medical condition if they answered "yes" to any of the following questions: "Have you ever been told by a doctor, nurse, or other health professional that you have COPD, emphysema, or chronic bronchitis; heart disease (angina or coronary heart disease, heart attack, or myocardial infarction); diabetes; or chronic kidney disease?" Respondent-reported height and weight were used to calculate BMI; respondents with $\mathrm{BMI} \geq 30 \mathrm{~kg}$ per $\mathrm{m}^{2}$ were considered to have obesity. A created variable captured persons having any of these conditions.

Nationwide estimates of underlying medical conditions were weighted to adjust for survey design. For county-level prevalence, estimates of each and of any condition were generated using a multilevel regression and poststratification approach (5) for 3,142 counties in all 50 states and DC. This approach has been validated in comparison with direct BRFSS survey estimates and local surveys for multiple chronic disease measures at state and county levels $(5,6)$. Briefly, a multilevel regression model was constructed for each outcome using individual-level age, 9 gender, race/ethnicity, ${ }^{* *}$ and educational-level ${ }^{\dagger \dagger}$ data

\footnotetext{
Age was categorized into 13 age groups at 5 -year intervals for ages $\geq 18$ years.

** Race/ethnicity was categorized as non-Hispanic white, non-Hispanic African American, non-Hispanic American Indian or Alaska Native, non-Hispanic Asian, non-Hispanic Native Hawaiian/other Pacific Islander, other single non-Hispanic race, two or more non-Hispanic race groups, and Hispanic.

$\dagger \dagger$ Education was categorized as less than high school, high school graduate, some college or technical school, or college graduate.
}

The MMWR series of publications is published by the Center for Surveillance, Epidemiology, and Laboratory Services, Centers for Disease Control and Prevention (CDC), U.S. Department of Health and Human Services, Atlanta, GA 30329-4027.

Suggested citation: [Author names; first three, then et al., if more than six.] [Report title]. MMWR Morb Mortal Wkly Rep 2020;69:[inclusive page numbers]

\section{Centers for Disease Control and Prevention \\ Robert R. Redfield, MD, Director}

Anne Schuchat, MD, Principal Deputy Director

Chesley L. Richards, MD, MPH, Deputy Director for Public Health Science and Surveillance

Rebecca Bunnell, PhD, MEd, Director, Office of Science

Arlene Greenspan, PhD, Acting Director, Office of Science Quality, Office of Science

Michael F. Iademarco, MD, MPH, Director, Center for Surveillance, Epidemiology, and Laboratory Services

Charlotte K. Kent, $\mathrm{PhD}$, MPH, Editor in Chief

MMWR Editorial and Production Staff (Weekly) Jacqueline Gindler, MD, Editor

Paul Z. Siegel, MD, MPH, Guest Associate Editor

Mary Dott, MD, MPH, Online Editor

Terisa F. Rutledge, Managing Editor

Douglas W. Weatherwax, Lead Technical Writer-Editor

Glenn Damon, Soumya Dunworth, PhD,

Teresa M. Hood, MS, Donald G. Meadows, MA Technical Writer-Editors
Michelle E. Bonds, MBA

Matthew L. Boulton, MD, MPH

Carolyn Brooks, ScD, MA

Jay C. Butler, MD

Virginia A. Caine, MD

\section{H}

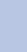

MMWR Editorial Board

Timothy F. Jones, MD, Chairman

Katherine Lyon Daniel, PhD

Jonathan E. Fielding, MD, MPH, MBA

David W. Fleming, MD

William E. Halperin, MD, DrPH, MPH

Jewel Mullen, MD, MPH, MPA Jeff Niederdeppe, $\mathrm{PhD}$

oyd, Lead Visual Information Specialist

Maureen A. Leahy, Julia C. Martinroe, Stephen R. Spriggs, Tong Yang, Visual Information Specialists

Quang M. Doan, MBA, Phyllis H. King,

Terraye M. Starr, Moua Yang,

Information Technology Specialists

Patricia Quinlisk, MD, MPH

Patrick L. Remington, MD, MPH

Carlos Roig, MS, MA

William Schaffner, MD

Morgan Bobb Swanson, BS 
from the 2018 BRFSS, and data on county-level percentage of the adult population living at $<150 \%$ of the poverty level from the 2014-2018 American Community Survey (ACS), a survey sent to about 3.5 million addresses each month that asks about topics not included on the decennial census, including education and employment. The model parameters were applied to 2018 Census county-level population estimates by age, gender, and race/ethnicity to calculate the predicted probability of each outcome. Because the U.S. Census Bureau does not provide county-level population data for education level by age, sex, and race/ethnicity, a bootstrapping approach ${ }^{\$ \S}$ was used to impute it. The estimated prevalence was obtained by multiplying the probability by the total population by county. Model-based estimates for any condition were validated by comparing them with the weighted direct survey estimates from counties with sample size $\geq 500$ (213) in BRFSS; the Pearson correlation coefficient was 0.89 . The county-level estimates of having any underlying medical condition were categorized into six county urban/rural classifications using CDC's National Center for Health Statistics definitions (large central metro/city, large fringe metro/suburb, medium metro, small metro, micropolitan, noncore/rural) (7). The overall weighted direct survey estimates were conducted using SUDAAN (version 11; RTI International), and other analyses were conducted using SAS (version 9.4; SAS Institute).

The nationwide prevalence of any of the five underlying medical conditions among adults aged $\geq 18$ years was $40.7 \%$ (95\% confidence interval $[\mathrm{CI}]=40.4 \%-41.0 \%)($ Table 1$)$. The overall weighted prevalences of these conditions were 30.9\% (obesity), $11.4 \%$ (diabetes), 6.9\% (COPD), 6.8\% (heart disease), and 3.1\% (CKD).

Among 3,142 counties, the median estimated (modeled) county prevalence of any underlying medical

\footnotetext{
$\overline{\$ \$}$ https://ww2.amstat.org/meetings/jsm/2016/onlineprogram/AbstractDetails. cfm?abstractid $=319359$.
}

condition was $47.2 \%$ (range $=22.0 \%-66.2 \%)$; obesity, $35.4 \%($ range $=15.2 \%-49.9 \%)$; diabetes, $12.8 \%$ (range $=6.1 \%-25.6 \%) ;$ COPD, $8.9 \%$ (range $=3.5 \%-19.9 \%)$; heart disease, $8.6 \%$ (range $=3.5 \%-15.1 \%$ ); and CKD, 3.4\% $($ range $=1.8 \%-6.2 \%)($ Table 1$)$.

Counties with the highest prevalences of any condition were concentrated in Southeastern states, particularly in Alabama, Arkansas, Kentucky, Louisiana, Mississippi, Tennessee, and West Virginia, as well as some counties in Oklahoma, South Dakota, Texas, and northern Michigan, among others (Figure) (Supplementary Table, https://stacks.cdc.gov/view/ cdc/90519). The estimated number of adults with any condition generally followed the population distribution, with higher estimated numbers of persons with any underlying medical conditions in more highly populated areas.

The estimated median prevalence of any condition generally increased with increasing rurality, ranging from $39.4 \%$ in large central metro counties to $48.8 \%$ in noncore counties (Table 2); the estimated median number of persons with any underlying condition ranged from 4,300 in noncore counties to 301,744 in large central metro counties.

\section{Discussion}

Three recent studies have reported that underlying medical conditions are highly prevalent among U.S. COVID-19 patients requiring hospitalization and ICU admission $(3,4,8)$. In this report, the median county prevalence of any of five underlying medical conditions that increase the risk for severe COVID-19-associated illness was 47.2\%, and prevalences were higher in counties in the southeastern United States and in more rural counties. These county level estimates can be used together with data on hospitalizations, ICU admissions, and ventilator use among COVID-19 patients with underlying conditions when planning for mitigation efforts and additional resource investment, including hospital beds, staffing, ventilators, and other medical supplies that might be needed

TABLE 1. Nationwide and model-based county-level $(n=3,142)$ estimates of prevalence and number of adults aged $\geq 18$ years with selected underlying medical conditions that might increase risk for severe COVID-19-associated illness — United States, 2018

\begin{tabular}{|c|c|c|c|}
\hline $\begin{array}{l}\text { Selected underlying } \\
\text { medical condition* }\end{array}$ & $\begin{array}{c}\text { Nationwide prevalence }{ }^{\dagger} \\
\%(95 \% \mathrm{Cl})\end{array}$ & $\begin{array}{c}\text { Median county prevalence } \\
\% \text { (range) }\end{array}$ & $\begin{array}{c}\text { Median county no. of adults }{ }^{\dagger} \\
\text { (range) }\end{array}$ \\
\hline Any & $40.7(40.4,41.0)$ & $47.2(22.0-66.2)$ & $9,743(41-2,877,316)$ \\
\hline Obesity $\left(\mathrm{BMI} \geq 30 \mathrm{~kg} / \mathrm{m}^{2}\right)$ & $30.9(30.6,31.2)$ & $35.4(15.2-49.9)$ & $7,174(25-2,097,906)$ \\
\hline Diabetes mellitus & $11.4(11.2,11.6)$ & $12.8(6.1-25.6)$ & $2,742(11-952,335)$ \\
\hline COPD & $6.9(6.7,7.0)$ & $8.9(3.5-19.9)$ & $1,962(7-434,075)$ \\
\hline Heart disease & $6.8(6.7,7.0)$ & $8.6(3.5-15.1)$ & $1,811(7-434,790)$ \\
\hline Chronic kidney disease & $3.1(3.0,3.3)$ & $3.4(1.8-6.2)$ & $717(3-237,766)$ \\
\hline
\end{tabular}

Abbreviations: $\mathrm{BMI}=$ body mass index; $\mathrm{Cl}=$ confidence interval; $\mathrm{COPD}=$ chronic obstructive pulmonary disease; COVID-19= coronavirus disease 2019 .

* Diabetes mellitus includes both type 1 and type 2 diabetes. COPD includes emphysema and chronic bronchitis. Heart disease includes angina or coronary heart disease, and heart attack or myocardial infarction.

† Weighted direct estimates from the Behavioral Risk Factor Surveillance System, 2018.

$\S$ Prevalence and number of adults estimated for 3,142 counties using a multilevel regression and poststratification approach applied to 2018 Behavioral Risk Factor Surveillance System data. 
FIGURE. Model-based estimates of U.S. prevalence (A) and number (B) of adults aged $\geq 18$ years with any selected underlying medical condition,* by county — United States, 2018

A

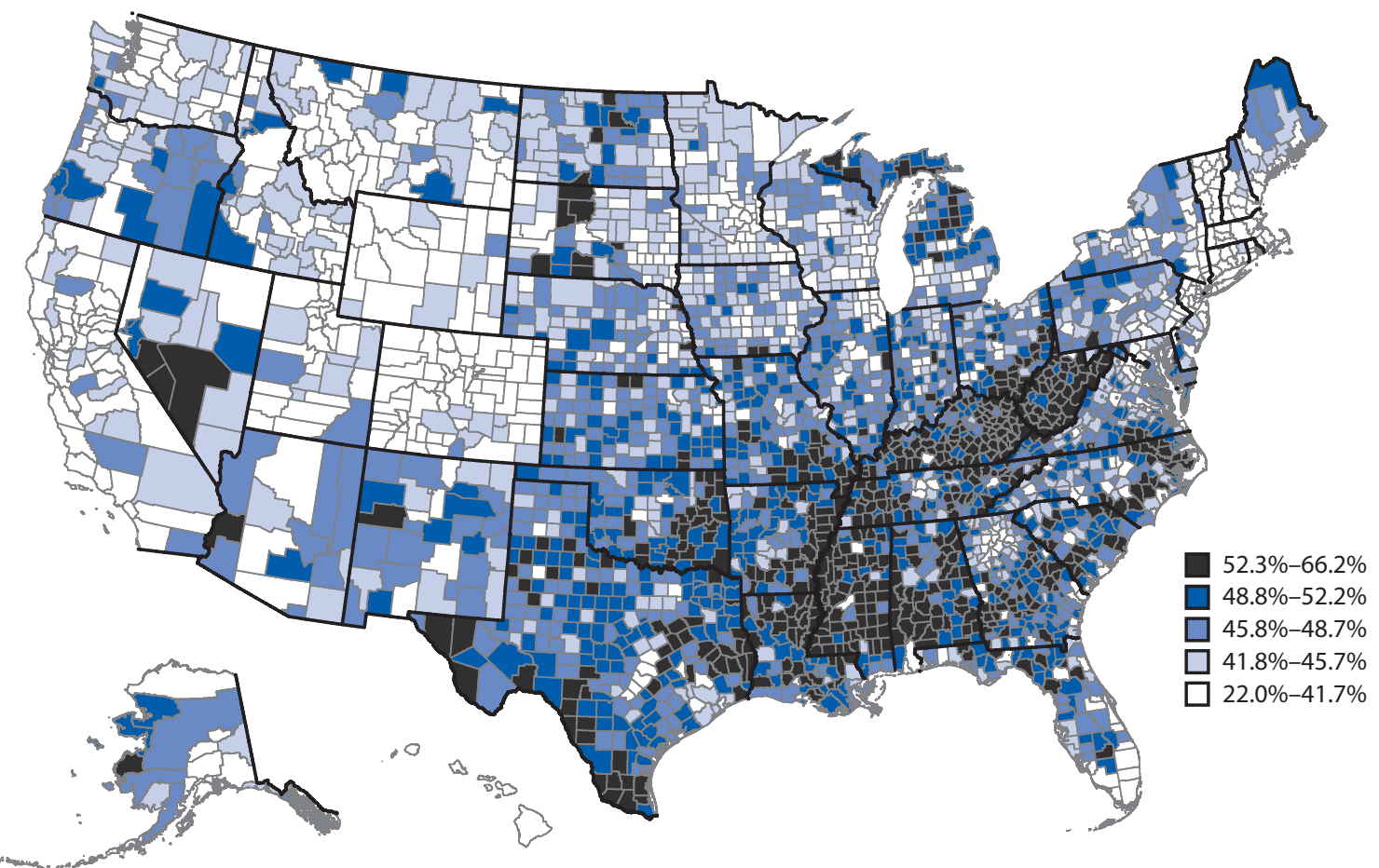

B



* Selected underlying conditions include chronic obstructive pulmonary disease, emphysema, or chronic bronchitis; heart disease (angina or coronary heart disease, heart attack, or myocardial infarction); diabetes; chronic kidney disease; or obesity (body mass index $\geq 30 \mathrm{~kg} / \mathrm{m}^{2}$ ). 
TABLE 2. Model-based estimates of prevalence and number of persons aged $\geq 18$ years with any select underlying medical condition, by urban/rural county classification — United States, 2018

\begin{tabular}{|c|c|c|c|}
\hline $\begin{array}{l}\text { County } \\
\text { classification* }\end{array}$ & $\begin{array}{l}\text { No. of } \\
\text { counties }\end{array}$ & $\begin{array}{c}\text { Median county } \\
\text { prevalence } \\
\% \text { (range) }\end{array}$ & $\begin{array}{l}\text { Median county } \\
\text { no. of } \\
\text { persons (range) }\end{array}$ \\
\hline \multicolumn{4}{|l|}{ Metropolitan } \\
\hline Large central metro $^{\dagger}$ & 68 & $\begin{array}{c}39.4 \\
(23.9-48.1)\end{array}$ & $\begin{array}{c}301,744 \\
(43,770-2,877,316)\end{array}$ \\
\hline Large fringe metro§ & 368 & $\begin{array}{c}43.9 \\
(26.4-56.9)\end{array}$ & $\begin{array}{c}34,221 \\
(1,611-725,284)\end{array}$ \\
\hline Medium metro" & 372 & $\begin{array}{c}45.5 \\
(22.0-61.7)\end{array}$ & $\begin{array}{c}33,687 \\
(659-332,209)\end{array}$ \\
\hline Small metro** & 358 & $\begin{array}{c}45.8 \\
(27.8-62.2)\end{array}$ & $\begin{array}{c}26,683 \\
(41-87,153)\end{array}$ \\
\hline \multicolumn{4}{|l|}{ Nonmetropolitan } \\
\hline Micropolitan ${ }^{\dagger \dagger}$ & 641 & $\begin{array}{c}47.8 \\
(24.3-64.6)\end{array}$ & $\begin{array}{c}13,979 \\
(176-59,820)\end{array}$ \\
\hline Noncore ${ }^{\S \S}$ & 1,335 & $\begin{array}{c}48.8 \\
(26.8-66.2)\end{array}$ & $\begin{array}{c}4,300 \\
(47-29,469)\end{array}$ \\
\hline
\end{tabular}

\footnotetext{
* Based on 2013 Urban-Rural Classification Scheme for Counties from the National Center for Health Statistics, CDC.

+ Large central metro counties in metropolitan statistical areas (MSAs) of 1 million population that 1) contain the entire population of the largest principal city of the MSA, or 2) are completely contained within the largest principal city of the MSA, or 3 ) contain $\geq 250,000$ residents of any principal city in the MSA.

$\S$ Large fringe metro counties in MSA of $\geq 1$ million population that do not qualify as large central.

ๆ Medium metro counties in MSA of 250,000-999,999 population.

** Small metro counties are counties in MSAs of $<250,000$ population.

t† Micropolitan counties in MSAs.

$\S \S$ Noncore counties not in MSAs.
}

to treat persons with underlying medical conditions, should they become ill with COVID-19.

The percentage of the population (prevalence) and the estimated numbers of adults with underlying medical conditions provide information for planning and have implications for health care resource utilization. Areas with comparatively lower prevalences but large populations, such as metropolitan areas, might still have large numbers of persons with underlying medical conditions at increased risk for severe COVID-19 illness. Conversely, areas with smaller populations but a comparatively higher prevalence of persons with underlying medical conditions might also have substantial need for additional resources to treat severe COVID-19 illness. Health care in rural counties is often underresourced, $\mathbf{9 9}$ and rural communities might have limited access to adequate care, which could further increase risk for poor COVID-19-associated outcomes. Prevalence estimates help highlight counties with a higher relative need for resources, whereas estimates of numbers of persons with underlying medical conditions help identify overall need by county; both can help decision-makers predict resource needs and develop resource allocation plans.

\footnotetext{
99 https://www.aha.org/system/files/2019-02/rural-report-2019.pdf.
}

\section{Summary}

What is already known about this topic?

Older adults and those with chronic obstructive pulmonary disease, heart disease, diabetes, chronic kidney disease, and obesity are at higher risk for severe COVID-19-associated illness.

What is added by this report?

The median model-based estimate of the prevalence of any of five underlying medical conditions associated with increased risk for severe COVID-19-associated illness among U.S. adults was $47.2 \%$ among 3,142 U.S. counties. The estimated number of persons with these conditions followed population distributions, but prevalence was higher in more rural counties.

What are the implications for public health practice?

The findings can help local decision-makers identify areas at higher risk for severe COVID-19 illness in their jurisdictions and guide resource allocation and implementation of community mitigation strategies.

The findings in this report are subject to at least five limitations. First, estimates were based on BRFSS data and subject to survey biases such as nonresponse, social desirability, and recall and knowledge of having a particular condition. Second, BRFSS data do not include all underlying medical conditions that might increase risk for severe COVID-19 illness, such as sickle cell disease, or information on organ transplant or disease severity. Third, some of the underlying medical conditions included in BRFSS might not exactly capture those conditions with the strongest and most consistent evidence such as specific heart conditions (e.g., cardiomyopathies and heart failure) or specific type of diabetes. Further, because COVID-19 is a novel disease and information regarding risk factors for severe illness is evolving, additional underlying medical conditions might be added in the future (as an example, cancer was added to the list after these analyses were conducted). Fourth, BRFSS data are collected for noninstitutionalized civilian persons and exclude populations that might be particularly vulnerable to severe COVID-19 illness, including those living in long-term care facilities and incarcerated populations, and might therefore not be representative for those groups. Finally, these estimates might be imprecise because of the multilevel regression modeling process and county-level population estimation.

These findings can be used by state and local decisionmakers to help identify areas at higher risk for severe COVID-19-associated illness because of underlying medical conditions and guide resource allocation and implementation of prevention and mitigation strategies. Future analyses could include weighting the contribution of each underlying medical condition according to the risk for severe COVID-19-associated outcomes, as well as identifying and 
incorporating other aspects of vulnerability to both infection and severe outcomes to better estimate the number of persons at increased risk for COVID-19. These findings highlight the prevalence of underlying medical conditions at the local (county) level that are important causes of morbidity and mortality on their own and increase risk for severe COVID-19-associated illness. These findings also emphasize the importance of prevention efforts to reduce the prevalence of these underlying medical conditions and their risk factors such as smoking, unhealthy diet, and lack of physical activity.

Corresponding author: Hilda Razzaghi, Hrazzaghi@cdc.gov.

${ }^{1}$ CDC COVID-19 Response Team; ${ }^{2}$ Division of Population Health, National Center for Chronic Disease Prevention and Health Promotion, CDC.

All authors have completed and submitted the International Committee of Medical Journal Editors form for disclosure of potential conflicts of interest. No potential conflicts of interest were disclosed.

\section{References}

1. Chow N, Fleming-Dutra K, Gierke R, et al.; CDC COVID-19 Response Team. Preliminary estimates of the prevalence of selected underlying health conditions among patients with coronavirus disease 2019-United States, February 12-March 28, 2020. MMWR Morb Mortal Wkly Rep 2020;69:382-6. https://doi.org/10.15585/mmwr.mm6913e2
2. CDC. Coronavirus disease 2019 (COVID-19). Evidence used to update the list of underlying medical conditions that increase a person's risk of severe illness from COVID-19. Atlanta, GA: US Department of Health and Human Services, CDC; 2020. https://www.cdc.gov/coronavirus/2019ncov/need-extra-precautions/evidence-table.html

3. Garg S, Kim L, Whitaker M, et al. Hospitalization rates and characteristics of patients hospitalized with laboratory-confirmed coronavirus disease 2019-COVID-NET, 14 states, March 1-30, 2020. MMWR Morb Mortal Wkly Rep 2020;69:458-64. https://doi.org/10.15585/mmwr. mm6915e3

4. Stokes EK, Zambrano LD, Anderson KN, et al. Coronavirus disease 2019 case surveillance-United States, January 22-May 30, 2020. MMWR Morb Mortal Wkly Rep 2020;69:759-65. https://doi.org/10.15585/ mmwr.mm6924e2

5. Zhang X, Holt JB, Lu H, et al. Multilevel regression and poststratification for small-area estimation of population health outcomes: a case study of chronic obstructive pulmonary disease prevalence using the behavioral risk factor surveillance system. Am J Epidemiol 2014;179:1025-33. https://doi.org/10.1093/aje/kwu018

6. Zhang X, Holt JB, Yun S, Lu H, Greenlund KJ, Croft JB. Validation of multilevel regression and poststratification methodology for small area estimation of health indicators from the behavioral risk factor surveillance system. Am J Epidemiol 2015;182:127-37. https://doi.org/10.1093/aje/ kwv002

7. Ingram DD, Franco SJ. 2013 NCHS urban-rural classification scheme for counties. Vital Health Stat 2 2014;(166):1-73.

8. Gold JAW, Wong KK, Szablewski CM, et al. Characteristics and clinical outcomes of adult patients hospitalized with COVID-19-Georgia, March 2020. MMWR Morb Mortal Wkly Rep 2020;69:545-50. https:// doi.org/10.15585/mmwr.mm6918e1 